\title{
Aspectos epidemiológicos e biopsicossociais em pacientes portadoras de câncer de mama
}

\author{
Epidemiological and Biopsychosocial aspects in patients with breast cancer
}

Aspectos espidemiológicos y biopsicosocialesen pacientes con cáncer de mama

Julia Rocha e Silva ${ }^{1}$, Ana Luísa Pereira Rodrigues ${ }^{1}$, Beatriz Emanuele da Silva Medeiros Guimarães $^{1}$, Gabriella Stéphany de Brito Teixeira ${ }^{1 *}$, Maria Clara Silveira Caixeta ${ }^{1}$, Sophia Queiroz Chaves Sibalszky¹, Priscila Capelari Orsolin¹, Natália de Fátima Gonçalves Amâncio¹.

\section{RESUMO}

Objetivo: Traçar o perfil epidemiológico das mulheres acometidas por câncer de mama no estado de Minas Gerais e identificar os prejuízos psicossociais ocasionados após o diagnóstico. Métodos: Estudo quantitativo, descritivo, do tipo transversal, com coleta de dados do Sistema de Informação do Câncer de Mama (SISMAMA) - DATASUS e no Sistema de Informação sobre Mortalidade (SIM), os quais foram projetados na plataforma Microsoft Excel, para confecção de tabelas e gráficos e posterior discussão de dados. Resultados: Traçou-se um perfil epidemiológico das mulheres mais acometidas por tal patologia- brancas, na faixa etária de 45 a 49 anos e com ensino fundamental incompleto - além de ter sido factível verificar como os aspectos biopsicossociais do câncer de mama podem interferir nos fatores prognósticos relacionados a essa neoplasia. Conclusão: A patologia neoplásica discutida no referido trabalho é muito incidente entre as brasileiras, o que demonstrou então a necessidade de maior atenção e cuidado no âmbito psicológico destas mulheres, aspecto esse que infere diretamente no discorrer do quadro prognóstico. Os resultados encontrados foram ao encontro das estatísticas previstas pelo Instituto Nacional de Câncer (INCA).

Palavras-chave: Câncer de mama, Impacto psicossocial, Mulheres.

\begin{abstract}
Objective: To trace the epidemiological profile of women affected by breast cancer in the state of Minas Gerais and to identify the psychosocial damage caused after diagnosis. Methods: Quantitative, descriptive, crosssectional study, with data collection from the Breast Cancer Information System (SISMAMA) - DATASUS and the Mortality Information System (SIM), which were designed on the Microsoft Excel platform, for making tables and graphs and later discussing data. Results: An epidemiological profile was drawn of the women most affected by such pathology - white, aged 45 to 49 years and with incomplete elementary education - in addition to being possible to verify how the biopsychosocial aspects of breast cancer can interfere in the prognostic factors related to this neoplasm. Conclusion: The neoplastic pathology discussed in that work is very common among Brazilian women, demonstrating then the need for greater attention and care in the psychological scope of these women, an aspect that directly inferred in the discussion of the prognostic picture. The results found were in line with the statistics provided by the National Cancer Institute (INCA).
\end{abstract}

Key words: Breast cancer, Psychosocial impact, Women.

${ }^{1}$ Centro Universitário de Patos de Minas (UNIPAM), Patos de Minas - MG.

*E-mail: gabriellastephany@unipam.edu.br

SUBMETIDO EM: 12/2020

ACEITO EM: 12/2020

PUBLICADO EM: 3/2021 


\section{RESUMEN}

Objetivo: Trazar el perfil epidemiológico de las mujeres afectadas por cáncer de mama en el estado de Minas Gerais e identificar el daño psicosocial causado tras el diagnóstico. Métodos: Estudio cuantitativo, descriptivo, transversal, con recolección de datos del Sistema de Información de Cáncer de Mama (SISMAMA) DATASUS y del Sistema de Información de Mortalidad (SIM), los cuales fueron diseñados en la plataforma Microsoft Excel, para la elaboración de tablas y gráficos y luego discutir los datos. Resultados: Se trazó un perfil epidemiológico de las mujeres más afectadas por dicha patología: blancas, de 45 a 49 años y con educación primaria incompleta - además de ser factible verificar cómo los aspectos biopsicosociales del cáncer de mama pueden interferir en los factores pronósticos relacionados con esta neoplasia. Conclusión: La patología neoplásica discutida en ese trabajo es muy común entre las mujeres brasileñas, demostrando la necesidad de mayor atención y cuidado en el campo psicológico de estas mujeres, aspecto que se infiere diretamente em la discusión del pronóstico. Los resultados encontrados coincidieron con las estadísticas proporcionadas por el Instituto Nacional del Cáncer (INCA).

Palabras clave: Cáncer de mama, Impacto psicosocial, Mujer.

\section{INTRODUÇÃO}

Os tumores malignos constituem a segunda maior causa de morte entre mulheres na idade reprodutiva, com incidência estimada de 2,2\% em mulheres na faixa etária de 14 a 40 anos, não se restringindo, entretanto, a esse grupo etário. Dentre neoplasias malignas, destaca-se o câncer de mama que é a segunda neoplasia mais frequente e segunda principal causa de morte por câncer no mundo e a primeira mais comum e principal causa de morte por câncer entre as mulheres do Brasil (GHONCHEH M, et al., 2016; ZUGAIB M, et al., 2016; INCA, 2020).

Assim como em outras neoplasias, o câncer de mama é uma proliferação clonal originada de células com várias mutações genéticas. Esse câncer pode ser do tipo hereditário - quando derivado da transmissibilidade de uma cópia anormal de um gene supressor tumoral, como o BRCA1, o BRCA2, o TP53 e o CHEK2 - ou esporádico - o qual apresenta como principal fator de risco a exposição hormonal aos estrogênios, uma vez que esses funcionariam como estimuladores do câncer de mama por meio de inúmeros mecanismos (KUMAR V, et al., 2016).

Aproximadamente metade das mulheres que são diagnosticadas com câncer de mama possuem fatores de risco identificáveis, além de faixa etária e sexo. A exposição ao estrogênio durante a vida, fatores reprodutivos, estilo de vida, fatores ambientais, antecedentes pessoais ou familiares de câncer de mama e história de doença benigna da mama também são considerados fatores que aumentam o risco para câncer de mama (CHABNER BA e LONGO DL, 2015).

A detecção precoce do câncer de mama é fundamental para garantir um tratamento mais efetivo e melhor qualidade de vida à paciente. Nesse viés, são realizados alguns testes genéticos para análise de possíveis mutações nos genes BRCA1 e BRCA2, indicados às pacientes cujo risco de ter uma mutação deletéria seja no mínimo de $10 \%$. Dessa forma, é possível que seja realizado um acompanhamento regular dessas mulheres e que haja uma maior chance de intervenção efetiva (CHABNER BA e LONGO DL, 2015).

Ademais, segundo Brasil (2010), o rastreamento para câncer de mama deve ser realizado a cada 2 anos, pelas mulheres sem histórico familiar dessa neoplasia, com idade entre 50 e 69 anos. O exame mais utilizado é a mamografia, avaliada pelo método de BIRADS, que analisa os riscos de haver uma neoplasia na região mamária.

O sistema BIRADS classifica as alterações mamárias em seis categorias de acordo com o risco de malignidade. Na categoria 0 encontram-se as mamografias de avaliação inconclusa, sendo necessária avaliação adicional com outro exame de imagem; na categoria 1, as mamografias em que não foram encontrados nódulos; na categoria 2, as mamografias de achados benignos, compreendendo os cistos simples, nódulos sólidos de características benignas e estáveis, entre outros (VASCONCELOS RG, et al., 2011). 
A categoria 3 descreve achados provavelmente benignos, com risco de malignidade de até $2 \%$, enquanto a categoria 4 descreve achados suspeitos, com risco de malignidade entre $3 \%$ e $94 \%$. Os achados altamente sugestivos de malignidade, ou seja, cujo risco de malignidade é superior a 95\%, são classificados na categoria 5 do sistema BIRADS. Por fim, a categoria 6 é destinada aos achados que definitivamente correspondem à câncer de mama, confirmado por exame histopatológico (VASCONCELOS RG, et al., 2011).

O léxico do BIRADS demonstra sua importância ao promover uma comunicação clara e precisa dos achados mamográficos, melhorando a assistência ao paciente e gerando novas oportunidades para garantia de qualidade, comunicação e pesquisa (TEIXEIRA MBR, 2011).

Devido ao fato de que a etiologia do câncer de mama abrange muitos fatores que, por sua vez, podem não ser modificáveis, sua prevenção torna-se dificultada. No entanto, é fundamental que haja um controle dos fatores de risco, estímulo aos fatores protetores e diagnóstico precoce. Logo, é fundamental evitar a obesidade e o consumo de bebidas alcoólicas, ter uma alimentação adequada e praticar exercícios físicos de forma regular. Outrossim, a amamentação pode ser considerada também como um fator protetor contra a neoplasia maligna da mama (BRASIL, 2019).

O diagnóstico de câncer de mama pode trazer inúmeras consequências à vida de uma mulher, uma vez que pode repercutir no âmbito físico, psicológico e social da paciente. É muito comum que diversos sentimentos venham à tona nesse momento, os quais podem contribuir negativamente para a evolução da neoplasia, sendo eles raiva, tristeza, ansiedade, medo e, até mesmo, o luto, já que são capazes de perturbar a homeostase do organismo (MIAJA M, et al., 2017).

O impacto do diagnóstico reflete diretamente na vida da paciente e também da família, ocasionando um momento de angústia e incertezas. Como a mama é um órgão que remete à feminilidade, a identidade da mulher é abalada e pode estar relacionada a perda do prazer, sensualidade e do afeto maternal. Sendo assim, ao longo do processo da doença a mulher enfrenta uma fase marcada pela necessidade do desapego da mama e aceitação da nova imagem (RAMOS BF, et al., 2013).

Com isso, de maneira mais explícita, é possível entender as diversas facetas de acometimento que um diagnóstico de câncer oferece a alguém, principalmente a ação do Câncer de Mama na vida de uma mulher, obrigando-a a ressignificar sua visão perante sua saúde, perante seus medos e perante seu corpo. Um diagnóstico como esse institui a essas mulheres a sensação de impotência, instaura sobre elas o medo da amputação, mais do que a amputação dos seios, a amputação da imagem socialmente aceita, a amputação de suas sexualidades e a amputação de um símbolo milenar de feminilidade (MATTIAS SR, et al., 2018).

Para entender de maneira mais clara o medo e o tabu da sociedade perante a neoplasia mamária é importante compreender melhor uma das vias de tratamento para os quadros em que o prognóstico já não é o mais positivo. O temor majoritário surge mediante a possibilidade da realização de uma mastectomia radical - tratamento cirúrgico agressivo que se baseia na ablação total das mamas -, procedimento que surgiu em meados do século XIX e que vem gradativamente sendo substituído por procedimentos mais conservadores, os quais tem como diferencial principal a preservação máxima das mamas de maneira segura, tanto para o prognóstico físico dessa paciente, quanto para melhor manutenção da integridade de sua saúde mental e autoimagem (ALMEIDA T, et al., 2012).

Vale-se também atribuir a ideia de que, mesmo com esse preceito social negativo em torno da cirurgia, muitas mulheres tentam superá-lo frente ao possível benefício que a mesma traria em suas vidas, mascarando assim toda sua insegurança e medos perante a possibilidade de cura. Entretanto, essas mesmas mulheres também necessitam de aporte médico, psicológico e familiar, porque, em algum momento, essa tentativa de negação e de superação é sobressaída pelo receio e pela falta de apoio em lidar com esse momento. (SILVA PLN, 2017)

Dessa maneira, compreende-se a necessidade de que essa paciente receba o apoio adequado da equipe médica e psicológica a partir do momento em que a mesma for diagnosticada e em que seu plano terapêutico comece a ser traçado. Para que, amparada, ela tenha total conhecimento de cada passo que será dado e assim, consiga lidar positivamente com o turbilhão de sentimentos gerados. Em vista disso, é válido citar que, 
por meio de estudos como o de Fornazari SA e Ferreira RER (2010), já é comprovado que a personalidade das mulheres perante a doença exerce interferência direita no enfrentamento do quadro; sendo assim, mulheres com maior autoconfiança, estabilidade emocional, pensamentos positivos e altos níveis de autoestima terão maior propensão ao bem-estar psicológico, o qual infere em um prognóstico mais bem quisto.

Diante do exposto, a importância do presente estudo justifica-se pela influência dos fatores psicológicos na evolução do câncer de mama. Portanto, o objetivo do presente estudo foi traçar o perfil epidemiológico das mulheres acometidas por câncer de mama no estado de Minas Gerais e identificar os prejuízos psicossociais ocasionados após o diagnóstico.

\section{MÉTODOS}

Trata-se de um estudo quantitativo, descritivo, do tipo transversal. Foi realizado um levantamento dos casos de câncer de mama registrados no Sistema de Informação do Câncer de Mama (SISMAMA), do Departamento de Informática do Sistema Único de Saúde (DATASUS), na região de Minas Gerais, no período de 2009 a 2013, visto que esse banco de informações só disponibilizava os dados até o ano de 2013. Foram coletados os dados referentes às variáveis: faixa etária, escolaridade, cor/raça, resultado da Punção Aspirativa por Agulha Fina (PAAF), localização primária do tumor e ano do diagnóstico.

Além disso, foram coletados dados acerca da mortalidade relacionada ao Câncer de mama, registrados no Sistema de Informação sobre Mortalidade (SIM), no período entre 2009 e 2018, período disponível e com informações mais atuais acerca de tais aspectos. Os dados pesquisados em tal plataforma são referentes à distribuição parcial do total de mortes femininas por câncer de mama em Minas Gerais e às taxas de mortalidade por câncer de mama na região Sudeste.

A posteriori, os dados foram alocados em uma tabela no Microsoft Excel, para confecção das tabelas e gráficos e para análise descritiva dos dados. $O$ trabalho foi realizado nas seguintes etapas: (I) pesquisa bibliográfica, (II) análise e coleta de dados a respeito dos casos de câncer de mama (III), cruzamento de dados entre as variáveis e casos de câncer de mama no estado de Minas Gerais entre 2009 e 2018.

Para desenvolvimento da pesquisa bibliográfica, a pergunta clínica "Como a jornada de enfrentamento do câncer de mama influencia as esferas biológicas, psicológicas e sociais das pacientes com tal patologia"? foi estruturada. Para elaboração da questão de pesquisa da revisão integrativa, utilizou-se a estratégia PICO (Acrômio para Patient, Intervention, Comparation e Outcome). Nela, temos $\mathrm{P}=$ mulheres acometidas com câncer de mama e $\mathrm{I}=$ influência da patologia nos aspectos biopsicossociais das pacientes.

A partir do estabelecimento das palavras-chave da pesquisa, foi realizado o cruzamento dos descritores "BreastCancer/Câncer de Mama", "Epidemiology/Epidemiologia"; "Risk Factors/Fatores de Risco"; "Pathophysiology/Fisiopatologia"; "Tracking/Rastreamento"; "Prevention/Prevenção"; "Psychosocial Consequences/Consequências Psicossociais"; nas seguintes bases de dados: Biblioteca Virtual de Saúde (BVS); National Library of Medicine (PubMed MEDLINE), Scientific Eletronic Library Online (SCIELO), EbscoHost, Medical LiteratureAnalysisandRetrieval System Online (Medline) e Google Scholar. A busca foi realizada nos meses de setembro e outubro de 2020. Foram considerados estudos publicados no período compreendido entre 2010 e 2020.

\section{RESULTADOS}

Utilizando-se dos meios de busca, principalmente do banco de dados DATASUS-TABNET, foi possível traçar um perfil das mulheres acometidas pela neoplasia mamária no estado de Minas Gerais, fornecendo então material para entrelace com os dados teóricos e subsequente construção epidemiológica dos dados mais importantes. Com isso, foi possível compreender de maneira mais clara os índices de acometimento, de morbimortalidade e de mortalidade, entender também as características físicas e sociais mais marcantes, as quais serão descritas a seguir. 
Tem-se a confirmação de que, no período transcorrido entre junho de 2009 a outubro de 2013, 13.518 mulheres foram diagnosticadas com algum grau de lesão neoplásica da mama no estado de Minas Gerais. Nota-se que o ano com maior incidência foi 2012, com 3.872 casos. Em 2012, a faixa etária mais acometida foi dos 45 aos 49 anos, com 617 casos. Contrapondo tais dados, o ano de 2009 apresentou o menor índice de notificações, com 912 casos, dos quais 158 ocorreram na mesma faixa etária citada anteriormente (Gráfico 1). De maneira geral, em todos os anos, entende-se que realmente a faixa etária dos 45 aos 49 anos é a mais comum durante o diagnóstico, com 2145 notificações totais (Gráfico 1).

Gráfico 1 - Distribuição, por faixa etária, dos achados histopatológicos de mulheres em Minas Gerais de junho/2009 a outubro/2013.

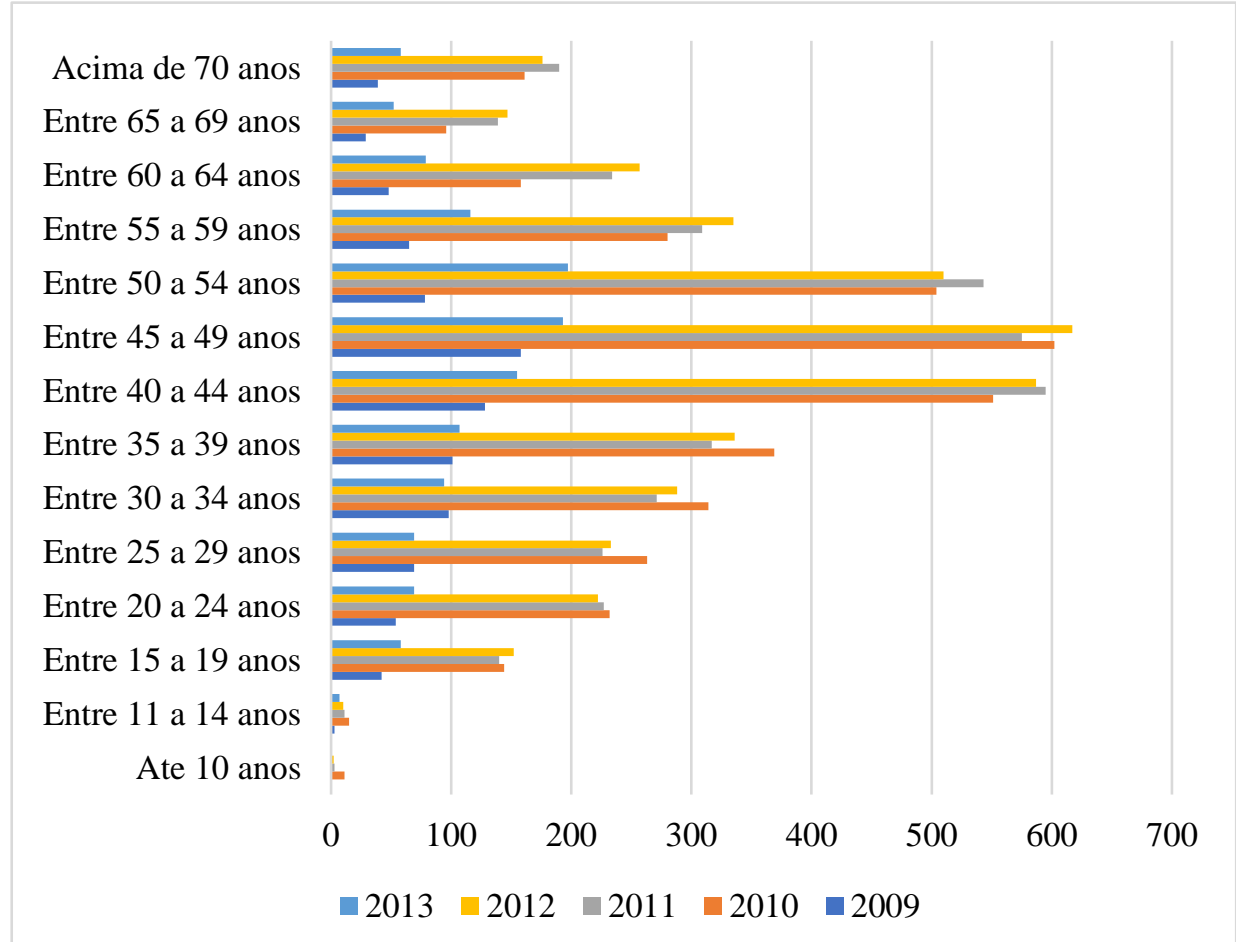

Fonte: Rocha e Silva J, et al., 2020; dados extraídos de Sistema de Informação do câncer e mama (SISMAMA).

Já em relação à escolaridade dessas mulheres que receberam o diagnóstico, percebe-se que a maioria delas - 3.051 ou $83,04 \%$ - ignoraram ou deixaram esta pergunta do questionário em branco. Entretanto, das 623 que responderam, 351 confirmaram ter ensino fundamental incompleto, frente há apenas 29 que afirmaram ter ensino superior completo (Tabela 1).

Tabela 1 - Achado histopatológico de lesão neoplásica maligna da mama, por escolaridade, em Minas Gerais, de junho/2009 a julho/2013.

\begin{tabular}{lc}
\hline Escolaridade & Lesão Neoplásica Maligna \\
\hline Ignorado/em branco & 3051 \\
Analfabeto & 69 \\
Ensino Fundamental Incompleto & 351 \\
Ensino Fundamental Completo & 125 \\
Ensino Médio Completo & 49 \\
Ensino Superior Completo & 29 \\
Total & 3674
\end{tabular}

Fonte: Rocha e Silva J, et al., 2020; dados extraídos de Sistema de Informação do câncer e mama (SISMAMA). 
Analisando-se as variáveis cor/raça e a incidência de câncer de mama em Minas Gerais, observa-se que, apesar de a maioria dos resultados positivos para malignidade, obtidos pelo exame de Punção Aspirativa por Agulha Fina (PAAF), não possuir informação quanto a cor/raça das pacientes $(88,86 \%)$, as mulheres brancas representam o grupo racial com maior concentração de resultados positivos para malignidade $(7,22 \%)$ (Tabela 2).

Tabela 2 - Distribuição dos resultados de PAAF positivos para malignidade conforme cor/raça, no estado de Minas Gerais, de junho/2009 a outubro/2013.

\begin{tabular}{lcc}
\hline \multicolumn{1}{c}{ Cor/Raça } & Positivo para malignidade & $\%$ \\
\hline Branca & 37 & 7,22 \\
Preta & 5 & 0,97 \\
Parda & 15 & 2,92 \\
Sem Informação & 455 & 88,86 \\
Total & 512 & 100 \\
\hline
\end{tabular}

Fonte: Rocha e Silva J, et al., 2020; dados extraídos de Sistema de Informação do câncer e mama (SISMAMA).

Somando-se a tais fatos e analisando os dados do Sistema de Informação sobre Mortalidade (SIM), é possível inferir também que de todas as mulheres mineiras que morreram em decorrência de câncer no período de 2014 a 2018, 15,22\% tiveram óbito em decorrência do câncer de mama. Porcentagem esta que sofreu elevação de 0,73\% quando comparada com os anos de 2009 a 2013 (Tabela 3).

Tabela 3 - Distribuição proporcional do total de mortes femininas por câncer de mama, segundo localização primária do tumor, em Minas Gerais, nos períodos de 2009-2013 e 2014-2018.

\begin{tabular}{ccc} 
Localizações primárias do câncer & $\mathbf{2 0 0 9 - 2 0 1 3}$ & $\mathbf{2 0 1 4 - 2 0 1 8}$ \\
\hline C50 - Câncer de mama & $14,49 \%$ & $15,22 \%$ \\
\hline
\end{tabular}

Fonte: Rocha e Silva J, et al., 2020; dados extraídos de MS/SVS/DASIS/CGIAE/Sistema de Informação sobre Mortalidade (SIM); MP/Fundação Instituto Brasileiro de Geografia e Estatística (IBGE); MS/INCA/Conprev/Divisão de Vigilância.

Por fim, é possível analisar detalhadamente as taxas - absolutas, brutas e ajustadas - de mortalidade por câncer de mama no Brasil, por cada ano, de 2009 a 2018. E com isso, é possível inferir que, em relação à taxa bruta, o ano com o maior número de mortes foi o de 2018, com taxa de 20,15 (Tabela 4).

Tabela 4 - Taxas de mortalidade por câncer de MAMA, brutas e ajustadas por idade, pelas populações mundial e brasileira de 2010, por 100.000 mulheres, Sudeste, entre 2009 e 2018.

\begin{tabular}{ccccc} 
Ano & Valor absoluto & Taxa bruta & Taxa ajustada mundial & Taxa ajustada Brasil \\
\hline 2009 & 6313 & 15,23 & 12,80 & 13,75 \\
2010 & 6641 & 16,08 & 12,85 & 13,77 \\
2011 & 6844 & 16,22 & 13,10 & 14,01 \\
2012 & 7033 & 16,53 & 13,03 & 13,97 \\
2013 & 7201 & 16,79 & 12,92 & 13,89 \\
2014 & 7445 & 17,23 & 13,02 & 13,96 \\
2015 & 7667 & 17,61 & 12,95 & 13,94 \\
2016 & 8094 & 18,59 & 13,72 & 14,74 \\
2017 & 8362 & 19,21 & 14,14 & 15,19 \\
2018 & 8774 & 20,15 & 14,76 & 15,9 \\
\hline
\end{tabular}

Fonte: Rocha e Silva J, et al., 2020; dados extraídos de MS/SVS/DASIS/CGIAE/Sistema de Informação sobre Mortalidade (SIM); MP/Fundação Instituto Brasileiro de Geografia e Estatística (IBGE); MS/INCA/Conprev/Divisão de Vigilância. 


\section{DISCUSSÃO}

Os dados obtidos nos resultados permitem concluir que, em Minas Gerais, no período entre junho de 2009 e outubro de 2013, a faixa etária mais acometida pelo câncer de mama foi das mulheres entre 45 e 49 anos, representando 15,86\%. Segundo o Instituto Nacional de Câncer (INCA), de 2020, a incidência deste tipo de câncer tende a crescer progressivamente a partir dos 40 anos.

Já em relação à escolaridade das mulheres que receberam o diagnóstico de câncer de mama, apesar de a grande maioria (83,04\%) não ter respondido o questionamento, um número expressivo de mulheres, 351 dentre as 623 que responderam, possuem ensino fundamental incompleto, enquanto que apenas 29 figuras femininas desse total possuem ensino superior completo. Isso pode ser explicado tendo em vista o acesso desigual a exames de rastreamento conforme o nível de instrução, sendo esse maior em populações com maior nível de escolaridade e renda (INCA, 2019).

A relação entre câncer de mama e etnia é bastante discutida na literatura. Kumar V, et al. (2018) relatam que, nos Estados Unidos, mulheres brancas não hispânicas têm maior incidência de tal patologia, no entanto, a população feminina hispânica e afro-americana tende a desenvolver esta condição em uma idade mais jovem e está mais suscetível a desenvolver tumores agressivos que se apresentam em estágio avançado. Do mesmo modo, dados do Grupo Brasileiro de Estudos do Câncer de Mama (SIMON SD, 2015) demonstram maior acometimento da etnia branca $(68 \%)$, seguida pela parda $(27,5 \%)$, negra $(3,9 \%)$, asiática $(0,5 \%)$ e indígena ( $0 \%$. Pode-se perceber, portanto, que os dados relativos ao acometimento de mulheres de diferentes raças em Minas Gerais seguem uma tendência nacional e até mesmo mundial.

No que tange aos aspectos incidência e mortalidade, o câncer de mama é o mais incidente em mulheres no mundo, representando $24,2 \%$ do total de casos em 2018, com aproximadamente 2,1 milhão de casos novos (INCA, 2020). Sendo assim, relacionando com os dados obtidos no estado de Minas Gerais é possível observar que a mortalidade das mulheres diagnosticadas com a neoplasia sofreu um aumento de $0,73 \%$, entre os períodos de 2009 a 2013 e 2014 a 2018, o que comprova os dados do INCA que afirmam que os óbitos por câncer de mama ocupam o primeiro lugar no país, representando $16,5 \%$ do total de óbitos no período de 2014-2018.

Ainda em relação a taxa de mortalidade por câncer de mama, de acordo com o INCA (2020), tendo em vista os dados da população mundial e também da população brasileira, é possível observar uma curva ascendente da incidência de mortes por tal neoplasia, representando a principal causa de mortes femininas brasileiras, com 13,84 óbitos/100.000 mulheres. As regiões Sudeste e Sul são as que apresentam as maiores taxas, com 14,76 e 14,64 óbitos/100.000 mulheres em 2018, respectivamente, o que pode estar relacionado a atuação de fatores de risco, como menor paridade, idade mais elevada no momento da primeira gestação e hábitos de vida que favorecem obesidade (CHABNER BA e LONGO DL, 2015; INCA, 2019).

Ademais, após traçar este perfil epidemiológico das mulheres que são acometidas pela neoplasia mamária, é de suma importância traçar também o perfil biopsicossocial das mesmas, buscando entender ao máximo como tal patologia interfere na vida delas e principalmente na forma com que elas observam sua autoimagem. Como já dito, na sociedade hodierna, o corpo funciona como um cartão de visita, uma maneira de inclusão na comunidade, preceito este, estipulado majoritariamente pela mídia associada à indústria da beleza. Entretanto, quando uma figura feminina enfrenta o câncer de mama, ela não está lidando apenas com o adoecer físico, mas também com o adoecer psicológico, com a forma de se ver enquanto mulher (ALMEIDA T, et al., 2012).

A neoplasia mamária e seus diversos tratamentos - mastectomia, quimioterapia, radioterapia - acarretam em desdobramentos negativos na forma com que a mulher se vê. Essas abordagens, quando não provocam mutilações à uma parte do corpo tão bem quista por essas mulheres - recursos cirúrgicos -, provocam efeitos colaterais avassaladores que geram desconforto, mudanças físicas e psicológicas, como a perda do cabelo, a fraqueza das unhas, náuseas e ganho ou perda de peso, sinais e sintomas estes que mudam totalmente a imagem que esta mulher tinha de si mesma, fazendo com que ela se sinta impotente perante sua feminilidade imposta socialmente (VIEIRA EM, et al., 2015). 
Dessa forma, havendo a percepção dos desdobramentos psicológicos desta patologia, foram desenvolvidas diversas escalas para melhor entender a forma com que as mulheres com câncer de mama se veem e enxergam seus corpos na tentativa de, criando um perfil epidemiológico, traçar políticas e ações efetivas ao tratamento multidisciplinar. Para esta neoplasia em específico usam-se 4 escalas principais: Body Image Index (BII), Sexual Adjustment and Body Image Scale (SABIS), Body Image after Breast Cancer Questionnaire (BIBCQ) e Body Image and Relationship Scale (BIRS) (VIEIRA EM, et al., 2015).

Tais tabelações possuem a capacidade de inferir sobre diversos quesitos na vida das figuras femininas, sendo os principais: imagem corporal, ajustamento sexual, vulnerabilidade, estigma corporal e limitações. E dessa maneira, é possível entender como tal distorção imagética acarreta no desenvolvimento de sintomas depressivos e quadros de ansiedade, demonstrando que não apenas a terapêutica medicamentosa para o próprio câncer é necessária para acolher tal mulher, mas principalmente o acolhimento psicológico assistido (PINTADO-CUCARELLA S e CHIBA-GIANNINI M, 2016).

Visto que a maioria dos sintomas que prejudicam a autoimagem feminina são desdobrados após o início dos tratamentos oncológicos, é valoroso que o acompanhamento psíquico destas mulheres se inicie antes, visando prepará-las perante tais intempéries e mostrando a elas maneiras que as capacitem para enfrentar as mudanças físicas e psicológicas que o câncer trará às suas vidas, mantendo o ideário de que elas continuam mantendo sua figura feminina mesmo diante tal situação (BINOTTO M e SCHWARTSMANN G, 2020).

\section{CONCLUSÃO}

Os resultados encontrados acerca do perfil epidemiológico das mulheres diagnosticadas com câncer de mama no Brasil e no estado de Minas Gerais foram ao encontro das estatísticas coletadas pelo Instituto Nacional de Câncer (INCA). Além disso, é muito evidente a relação entre o diagnóstico de tal patologia e certas alterações psicológicas nessas pacientes, o que pode ser extremamente prejudicial à qualidade de vida e ao estado emocional. Assim, torna-se imprescindível que essas mulheres tenham o mais alto nível de apoio e de atenção da equipe médica e da equipe psicológica. Ademais, é essencial que a realização de novas pesquisas sobre a temática dos aspectos biopsicossociais no câncer de mama seja estimulada, o que poderia possibilitar um melhor entendimento acerca dessa questão no contexto brasileiro.

\section{REFERÊNCIAS}

1. ALMEIDA T, et al. Repercussões do câncer de mama na imagem corporal da mulher: uma revisão sistemática. Physis Revista de Saúde Coletiva, 2012; 22(3): 1003-1029.

2. BINOTTO M, SCHWARTSMANN G. Qualidade de Vida Relacionada à Saúde de Pacientes com Câncer de Mama: Revisão Integrativa da Literatura. Revista Brasileira de Cancerologia, 2020; 66(1), 1-12.

3. BRASIL. Ministério da Saúde. Secretaria de Atenção à Saúde. Departamento de Atenção Básica. Rastreamento / Ministério da Saúde, Secretaria de Atenção à Saúde, Departamento de Atenção Básica. - Brasília: Ministério da Saúde, 2010.

4. BRASIL. 2019. In: Ministério da Saúde. Outubro Rosa - Mês de conscientização sobre o câncer de mama. Biblioteca Virtual em Saúde. Disponível em: http://bvsms.saude.gov.br/component/content/article?id=30 41. Acesso em: 20 nov. 2020.

5. CHABNER BA, LONGO DL. Manual de oncologia de Harrison. 2 ed. Porto Alegre: AMGH Editora Ltda, 2015; $936 \mathrm{p}$.

6. FORNAZARI SA, FERREIRA RER. Religiosidade/espiritualidade em pacientes oncológicos: qualidade de vida e saúde. Psicologia: Teoria e Pesquisa, 2010; 26(2).

7. GHONCHEH M, et al. Incidence and Mortality and Epidemiology of Breast Cancer in the World.Asian Pacific JournalofCancerPrevention, 2016; 17(3): 43-46.

8. INSTITUTO NACIONAL DE CÂNCER JOSÉ ALENCAR GOMES DA SILVA. A situação do Câncer de Mama no Brasil: Síntese de Dados dos Sistemas de Informação. Rio de Janeiro: INCA, 2019. Disponível em: $<$ https://www.inca.gov.br/sites/ufu.sti.inca.local/files/media/document/a_situacao_ca_mama_brasil_2019.pdf>. Acesso em: 14 set. 2020.

9. INSTITUTO NACIONAL DE CÂNCER JOSÉ ALENCAR GOMES DA SILVA. Informativo Vigilância do Câncer. Rio de Janeiro: $\quad 2020 . \quad$ Disponível em: <https://www.inca.gov.br/sites/ufu.sti.inca.local/files//media/document//informativo-vigilancia-do-cancer-n8-2020.pdf>. Acesso em: 29 out. 2020.

10. KUMAR V, et al. Robbins \& Cotran Patologia - Bases Patológicas das Doenças. 9. ed. Rio de Janeiro: Elsevier Editora Ltda, 2016; 421p. 
11. MATTIAS SR, et al. Câncer de mama: sentimentos e percepções das mulheres diante do diagnóstico. Revista Online de Pesquisa: Cuidado é fundamental, 2018; 10(2): 385-390.

12. MIAJA M, et al. Psychological Impact of Alterations in Sexuality, Fertility, and Body Image in Young Breast Cancer Patients and Their Partners. Revista de Investigación Clínica, 2017; 69: 204-209.

13. PINTADO-CUCARELLA S, CHIBA-GIANNINI M. Alteraciones de la autoestima y la imagen corporal y síntomas de depresión y ansiedad en una muestra de pacientes mexicanas con cáncer de mama. salud pública de México, 2016, 58(4): 400-401.

14. RAMOS BF, et al. Câncer de mama feminino e psicología. Revista Sociedade Brasileira de Psicologia Hospitalar, 2013, 59 (3): 351-359.

15. SILVA PLN, et al. Impacto de la mastectomía sobre la vida sexual y emocional de las mujeres atendidas por un servicio de salud del norte de Minas Gerais. Cultura de los Cuidados, 2017; 49.

16. SIMON SD. Grupo Brasileiro de Estudos em Câncer de Mama (Gbecan). Projeto Amazona. Dados preliminares de câncer de mama no Brasil [Internet]. Disponível em: <http://www.gbecam.org.br/downloads/Projeto_Amazona.pdf>. Acesso em 29 out. 2020.

17. TEIXEIRA MBR. Avaliação dos achados mamográficos classificados na categoria 4 do sistema BI-RADS e sua correlação histopatológica. Dissertação para obtenção do título de Mestre - Faculdade de Medicina de Botucatu, Universidade Estadual Paulista. Botucatu, 62p, 2011.

18. VASCONCELOS RG, et al. Ultrassonografia mamária - Aspectos contemporâneos. Revista Comunicação em Ciências da Saúde, 2011, 22(1): 129-140.

19. VIEIRA EM, et al. Validação do Body Image Relationship Scale para mulheres com câncer de mama. Revista Brasileira de Ginecologia e Obstetrícia, 2015; 37(10): 473-479.

20. ZUGAIB M, et al. Zugaib obstetrícia. 3a ed. Barueri: Manole Ltda, 2016; 1330p. 